\title{
Palm oil, its nutritional and health implications (Review)
}

\section{*11MOISI, OB; ILORI, GE; AGHO, I.; EKHATOR, JO}

\author{
Nigerian Institute for Oil Palm Research, P.M.B 1030, Benin-City, Edo State. Nigeria \\ E-mail of authors;-imoisib@gmail.com, esohe_grc@yahoo.com,imagho@yahoo.com and jeff_yahoo.com
}

KEYWORDS: Oxidized, Saturated Fats, Palm Oil, Coronary Heart Disease.

\begin{abstract}
Diet for some time now in Nigeria has undergone many changes such as changes in dietary intake of fats and oils. There has been an increasing consumption of partially hydrogenated trans-vegetable oils and a decreasing intake of lauric acid-containing oils. Although popular literature and people generally, usually attribute an increased risk of coronary heart disease (CHD) to elevated levels of serum cholesterol, which in turn are thought to derive from an increased dietary intake of saturated fats and cholesterol. The palm oil and palm kernel oil are high in saturated fatty acids, about 50\% and $80 \%$ respectively and are esterified with glycerol. In developing countries, vegetable oils are replacing animal fats because of the cost and health concerns. It is reassuring to know that the consumption of palm oil as a source of dietary fat does not pose any additional risks for coronary artery disease when consumed in realistic amounts as part of a healthy diet. However, oxidized palm oil induces reproductive toxicity and organ toxicity particularly of the kidneys, lungs, liver and heart. Therefore, oxidized palm oil should be avoided.

(C) JASEM
\end{abstract}

\section{http://dx.doi.org/10.4314/jasem.v19i1.17}

\section{Introduction}

Palm oil and palm kernel oil are edible plant oils derived from the fruits of palm trees. Palm oil is extracted from the pulp of the fruit of oil palm (Elaeis guineensis); palm kernel oil is derived from the kernel seed of the oil palm (Poku, 2002). Palm oil is naturally reddish in colour because it contains a high amount of beta-carotene. Palm oil has been used in food preparation for over 5,000 years. Palm oil is the most widely produced edible vegetable oil in the world and its nutritional and health attributes have been well documented (Chandrasekharan et al. 2000).

According to the Malaysian Oil Palm Statistics in 2005

//www.tocotrienol.org/en/index/news/58.html), it surpassed soybean oil as the most widely produced vegetable oil in the world. Palm oil is currently enjoying strong appeal worldwide as a cooking aid because it is free of Artery-clogging trans-fats. Besides being cleaner and more stable, it is consumed worldwide as cooking oil, in making of margarine and shortening, apart from being used as an ingredient in fat blends and a vast array of food products. In the United States, Palm oil's principal edible use is as an ingredient in prepared foods (primarily baked foods). Food manufacturers choose palm oil because it has a distinct quality, requires little or no hydrogenation, and prolongs the shelf life of different products (Anonymous 2003). Malaysia and Indonesia account for 83 percent of production and 89 percent of global exports.

Palm Oil Composition of Palm Oil: Palm oil and palm kernel oil are high in saturated fatty acids; palm oil is semi solid at room temperature. Palm oil contains several saturated and unsaturated fats in the forms of glyceryl laurate $(0.1 \%$ saturated), myristate ( $1 \%$, saturated), palmitate ( $44 \%$, saturated), stearate (5\% saturated), oleate (39\% monounsaturated), linoleate (10\% polyunsaturated) and linolenate $(0.3 \%$ polyunsaturated) (Cottrell, 1991). Palm kernel oil is highly saturated. Palm kernel oil contains mainly lauric acid. Palm oil is the largest natural source of tocotrienol. The development of convenience foods and of snack food manufacture on an industrial scale opened up additional uses for palm oil, because of its good resistance to oxidative deterioration and its better ability to withstand the high temperatures used in frying than most alternative oils (Kheri, 1987; Berger, 1992)

Antioxidants in palm oil: Effects of carotenoids: Red palm oil gets its characteristics dark red colour, which comes from carotenes such as alpha-carotene, beta-carotene and lycopene. The same nutrient that give tomatoes, carrots and other fruits and vegetable their rich colours. Crude palm oil is considered to be 
the richest natural source or carotenoids also enhance immune function by a variety of mechanisms, and can improve cardiovascular health. Carotenoids also play an important potential role by acting as biological antioxidants, protecting cells and tissues from the damaging effect of free radicals. When on being exposed to pollutants in cigarette smoke, industrial pollution, stress, unbalance diets, pesticide and insecticide residues in food and water, and many other negative environmental influences, one is also exposed to free radicals. A build-up of free radicals in the body is associated with degenerative diseases such as heart disease and cancer, as well as general ageing. It is, therefore, in one's own best interest to ensure that to eat a diet rich in antioxidants that will prevent the damage that is done to bodies by free radicals. Red palm oil is a form of processed palm oil retains $80 \%$ of the original carotenoids, making it a remarkable source of Vitamin A. These natural antioxidants act as buffers against free radicals and are believed to play a protective role in cellular ageing, atherosclerosis, cancer, arthritis, and Alzheimer's disease. (Sutapa and Analava, 2009).

Effects of Tocopherols and Tocotrienol: The tocopherol is one of the most interesting features in palm oil because it consists mainly of the tocotrienol, with an unsaturated side chain. These are not found in other common vegetable oil. Analytical work has shown that an average of 50 to 60 percent of the tocopherol content remains after refining conditions used. They are less volatile and therefore, more persistent in high temperature conditions, such as in deep fat frying. The tocopherol content is a major fact in stabilizing palm oil against oxidation. In fact, no other vegetable oil has as much Vitamin $\mathrm{E}$ as compared to palm oil (Chow 1992). Palm oil contains between 500 to $800 \mathrm{ppm}$ tocopherols which are antioxidants. Therefore, crude palm oil is stable antioxidants and does not need extra. Natural vitamin $\mathrm{E}$ exists in eight different forms or isomers, four tocopherols and four tocotrienols. Natural palm oil contains alpha, beta and gamma. Tocotrienols in Vitamin E have been found to have antioxidant and anti-cancer activities. Red palm oil antioxidant like tocotrienols and carotenes are added to foods and cosmetics due to their purported health benefits (Stuchlik, 2002; Rona, 2004). Red palm oil has been blended into mayonnaise and salad oil (Nagendran, 2000). Tocotrienols by its action on liver enzymes lowers blood cholesterol level without reduction in good cholesterol (High Density Lipoprotein, HDL). Its antioxidant properties bring many benefits to the human body, such as preventing skin aging, preventing fat oxidation, reducing blood pressure etc. Human studies have shown that palm tocotrienols have the ability to reverse blockage of the carotid artery and platelet aggregation (the clumping together of cells) thereby reducing the risk of stroke, arteriosclerosis, and ischaemic heart diseases. Palm tocotrienols have been shown to be protective after a strenuous bout of exercise by preventing protein oxidation and lipid peroxidation. Tocotrienol-rich fraction of palm oil is capable of protecting brain against oxidative damage and thereby ensuing adverse alterations that accompany aging. Lipid peroxides in blood vessels and plasma show a positive correlation with blood pressure. The antioxidant ability of gamma-tocotrienol may prevent development of increased blood pressure by reducing lipid peroxides and enhancing the total antioxidant status, Melanoma, also on the increase, can be inhibited with the delta fraction of tocotrienols. When applied topically, vitamin E tocotrienols is quickly absorbed into the deep layers of the skin.

Gamma- and delta-tocotrienols derived from palm oil exhibit a strong activity against tumor promotion. The delta and gamma factions of tocotrienols can inhibit certain types of cancer, including both the estrogen-positive and estrogen-negative breast cancer cells. The inhibition of the growth of breast cancer cells by palm tocotrienols could have extraordinarily important clinical implications on world health. Not only can the palm tocotrienols prevent the growth of these unwanted cells, but they can also do this in the presence as well as in the absence of this estradiol, thereby protecting against both hormone-related and other kinds of breast cancer. It is interesting to note that tocotrienols can inhibit or even kill normal cells, but only in extremely high amount.

Fatty acids: The fatty acids composition of palm oil is compared with other major oils and fats in their natural state i.e unhydrogenated. The major difference between palm oil and other oils and fats is its higher proportion of palmitic acid in the constituent fatty acids. Palm olein and palm stearin share the same major fatty acids, namely palmitic acid, oleic acid and linoleic acid. However, palm olein has relatively more oleic acids and linoleic and less palmitic acids than does palm stearin. Palm oil has a semisolid texture at room temperature, so hydrogenation is not necessary. Other oils, such as soybean or rapeseed, are hydrogenated during the manufacture of margarines and shortenings. Hydrogenated fats often contain appreciable proportions of trans fatty acids and the effect of excessive intakes of these on health is uncertain (British Nutrition Foundation, 1987). 
The concentration of fatty acids at the triglyceride 2position of a fat has dietary consequences. Palm oil has more palmitic acid at the 2-position than does any other common vegetable oil and it was shown that infants thrive on feeds with an enriched content of palmitic acid at the triglycerides 2-position (Droese, et al; 1976). With regards to triglycerides, palm oil is distinguished from other oils by its high levels of 48 , 50 and 52-C glycerides. The 50- and 52-C glycerides are present at almost equal levels.

Minor constituents: Although palm oil contains tocopherols, tocotrienols and carotenoids. There are other minor components such as-;

Sterols: Sterol in palm oil does not seem to serve any useful function in the oil nor do they have any detrimental effect (Goh, et al; 1985).

Polar lipids: The main phospholipid in palm oil is phosphatidycholine and the major glycolipid is monogalactosyldiglyceride. The wet milling process leaves most of the phospholipids in the palm fruit, so crude palm oil contains relatively low levels of phospholipids $(5-130 \mathrm{mg} / \mathrm{L})$. It has been reported that most of the phosphorus in the oil is present as inorganic phosphate, which may be undesirable. On the other hand, phospholipids stabilize colloidal dispersions and can be described as antioxidant synergists (Goh, et al; 1985).

Phenols: During frying, palm oil tends to darken initially more quickly than some other oils and this may be due to the presence of phenolic compounds. Total concentration is usually $<100 \mathrm{mg} / \mathrm{L}$ in crude palm oil (Berger, 1987).

Free fatty acids.: Free fatty acids are formed as a result of hydrolysis of triglycerides. Crude palm oil normally contains $2.5-5 \%$ of free fatty acids when it reaches the refinery but badly handled oils may contain up to $10 \%$ (Young, 1987).

Impurities: Small amount of iron and copper may be present in the crude oil, which are pro-oxidation catalytic amounts. Iron impurities are derived from wear and tear of oil mill machinery and may be minimized by using stainless steel at strategic points in the milling process or by magnetic traps and filters. Crude palm oil usually contains $<5 \mathrm{ug} / \mathrm{L}$ iron when these precautions are taken. Other possible impurities include tannins and trace flavonoids (Goh, et al; 1985).

Fatty acids compositions of Palm kernel Oil (Chow 1992) Palm Kernel Oil Saturated acids-Lauric $\left(C_{12}\right)$
48.2\%, Myristic $\left(\mathrm{C}_{14}\right)$ 16.2\%, Palmitic $\left(\mathrm{C}_{16}\right)$ 8.4\%, Capric $\left(\mathrm{C}_{10}\right) 3.4 \%$, Caprylic $\left(\mathrm{C}_{8}\right) 3.3 \%$, Stearic $\left(\mathrm{C}_{18}\right)$ $2.5 \%$.

Mono Unsaturated acids-Oleic $\left(\mathrm{C}_{18}\right) \quad 15.3 \%$ Poly Unsaturated acids-Linoleic $\left(\mathrm{C}_{18}\right) 2.3 \%$ Other/Unknown 0.4\%. Akintayo and Bayer (2002) reported that the free fatty acid composition of palm kernel oil values are above the allowable limits, which may make it undergo oxidation and may easily become rancid. Free fatty acid and acid value of PKO are high, indicating that they would require refining to make them suitable for edible purposes, and may be better utilized for industrial purposes in crude state.

Food uses of palm oil: Characteristics of palm oil and its fractions: Palm oil processes several characteristics that are important in determining its incorporation into food products (Kheiri, 1987). 1). It has high solid-glyceride content, giving the required consistency without hydrogenation. 2). It is very resistant to oxidation and therefore has a long shelf life. 3). Its level of high melting point triglycerides together with its relatively low solid content at $10^{\circ} \mathrm{C}$ helps in formulation of products with a wide plastic range, which are suitable for hot climates and some industrial applications. 4). It has the tendency to crystallize in the small beta prime crystals, a property desirable for some applications, e.g for margarine and cakes. 5). Its prices are often competitive. 6). Because of its linoleic acid content (10-11\%) it can only be used in limited quantities in margarines specifying a high PUFA level. 7). It has relatively slow melting properties because of the wide plastic range. 8). Its slow crystallization properties can lead to structural hardness in the finished product and a tendency for recrystallization.

Margarines: One of the major uses of palm oil and its products is in margarine. Palm olein is suitable as the liquid component of margarine blends, particularly in the firmer grades of products. Palm stearin is of some value as a hard stock but at higher levels it tends to cause posthardening. It can be interesterfied with olein and then used in plastic shortenings (Traitler, 1985).

Shortening: These are semisolid fats very similar in function and formulation to cake margarines, but moisture free. They impart an easily broken, crumbly texture, which melts in the mouth, to biscuits and short pastry. Consistency and spreadability of the fat are important with a soft, smooth consistency needed. Blends based on palm oil, hydrogenated palm oil, or 
blends of palm oil and palm stearin are widely used (Berger, 1987).

Vanaspati: This is a semisolid granular product, similar in formulation to the bakery shortenings, mainly used as a general purpose cooking fat. It may be regarded as a vegetable ghee, having the same relationship to ghee that margarine has to butter. Vanaspati is usually formulated from partly hydrogenated soya, cottonseed or rapeseed oils together with up to $80 \%$ palm oil (Berger, 1987).

Frying fats: Palm oil and olein have good oxidative stability due to the presence of natural antioxidants (tocopherol and tocotrienols) and the absence of linoleic acid. They are comparatively cheap to use and produce fried food products with good flavor and long shelf life (Berger, 1987). Palm olein was found to compare well with groundnut oil and deteriorates less rapidly than many other vegetable oils such as sunflower oil and hardened soybean oil (Bracco, 1985). However, on repeated frying, a brown colour is formed from phenolic minor components in palm oil products and this colouration is unrelated to the deterioration of the fat.

Confectionery fats: Palm midfraction is used as a coco-butter extender or as the main component (50$70 \%$ ) of coco-butter equivalent. This fraction should exhibit properties similar to those of cocoa butter (Deffense, 1985). Two product based on hydrogenated palm olein have also been used as cocoa butter extenders (Berger, 1986).

Other uses: Hard palm stearin is used as a dough improver, as a crystallization starter in the confectionary industry and after glycerolysis, in food emulsifier preparations (Traitler, 1985). Stearin and hydrogenated palm oil are also used in dried soups and powder mixes (Kheiri, 1987). Olein when mixed with other fats and oils, gives a suitable fat mix for baby-food formulations (Traitler, 1985). Together with palm oil, it is also used in filled milk and liquid coffee whiteners. Palm oil, on its own or blended with palm kernel oil refined, can also be used in nondairy ice cream, chocolate coatings, and sandwich cream. (Kheiri, 1987).

Benefits of Palm Oil: Palm oil is consumed in the fresh state and or at various levels of oxidation. Feeding experiments in various animal and humans highlighted the beneficial role of fresh palm oil to health. Although when obtained in the crude state, oils and fat are mainly unpalatable, but the health benefits include reduction in the risk of arterial thrombosis and atherosclerosis, inhibition of cholesterol biosynthesis and platelet aggregation, and reduction in blood pressure. However, on being used in the oxidized state possesses potential dangers to the physiological and biochemical functions of the body. Oxidized palm oil induces an adverse effect on plasma lipid profile, free fatty acids, phospholipids and cerebrosides. Additionally, oxidized palm oil induces reproductive toxicity and organ toxicity particularly of the kidneys, lungs, liver and heart. Available evidence suggests that at least part of the oxidized oil impact on health is due to generation of toxicants due to oxidation. Therefore, oxidized oil and or the level of oxidation may reduce the health risk (Ebong et al; 1999). A study by a group of researchers in China comparing palm, soybean, peanut oils and lard showed that palm oil actually increased the levels of good cholesterol and reduced the levels of bad cholesterol in the blood (Zhang, et al. 1997 cited by Koh, 2007). When not oxidized. A study by Hornstra in 1990 also showed similar results. Zhang, et al. (1997) found that in normal and hypercholesterolemic subjects, the use of palm oil in the diet should be safe and will not increase the risk of Cerebro Vascular Disease (CVD). Toxicological and pharmacological studies show that supplementation with palm tocotrienols up to 2,500 milligrams per day per kilogram of body weight does not produce any significant side effects. Although higher levels can be used for therapeutic purposes, those who want to enhance their Antioxidant intake can use 30 to 50 milligrams of tocotrienols daily (http://www.bettykamen.com/newsletters/palmoil.ht m).

Controversies of Palm Oil: For many years now, it has been established that the primary cholesterolelevating fatty acids are the saturated fatty acids with 12 (lauric acid), 14 (myristic acid) and 16 (palmitic acid) carbon atoms with a concomitant increase in the risk of coronary heart disease. The World Health Organization in its report (2003) states that there is convincing evidence that palmitic oil consumption contributes to an increased risk of developing cardiovascular diseases. In the past, palm oil was attacked as "saturated" since it contains $44 \%$ palmitic acid and 5\% stearic acid, and thereby allegedly raises blood cholesterol and increases the risk of cardiovascular disease. However, people even in hospitals were advised to consume little palm oil. But, a sizeable and growing body of scientific evidence indicates that palm oil's effect on blood cholesterol is relatively neutral when compared to other fats and oils. Palm oil raises plasma cholesterol only when an excess of dietary cholesterol is presented in the diet. Two main meta-analyses have examined the effect of palmitic acid (found in palm 
oil) on serum cholesterol. In a 1997 study based on 134 clinical studies, British researchers concluded that, compared to carbohydrates, palmitic acid raises blood cholesterol levels (Clarke, et al. 1997). In 2003, Dutch scientists conducted another metaanalysis of 35 clinical studies (Mensink, et al. 2003) and Examined what many experts consider the best indicator of heart-disease risk: the ratio of total Cholesterol to HDL cholesterol (Institute of Medicine, National Academies 2002). Palmitic acid increased the total: HDL cholesterol ratio more than other saturated fatty acids, including lauric acid and mysristic acid, which are abundant in palm kernel oil and coconut oil; the other highly saturated tropical oil (Enig, 1993). Palm oil increases the total: HDL cholesterol ratio more than the average U.S. or British dietary fat (Jensen, et al. 1999). That finding indicates that, in terms of blood cholesterol, palm oil is somewhat more harmful than the average U.S. dietary fat and much more harmful than such liquid oils as olive, soy and canola. The World Health Organization has stated that there is "convincing evidence" that palmitic acid increases the risk of cardiovascular disease (World Health Organization 2003). But due to the antioxidant present it neutralizes the palmitic acid present in it. But if the Palm Oil is oxidized and the Free Fatty Acids is more than $5 \%$ it becomes dangerous, a number of pre-1990 human feeding studies. Reported that palm oil diets resulted in lower serum cholesterol levels than prestudy values. Indeed, scientists concluded that these studies, although not specifically designed to study palm oil, have revealed that a palm oil diet lowered plasma cholesterol compared with the starting periods during which the subjects were eating their habitual Western diets. These conclusions were questioned because the studies were not designed to measure the effects of palm oil. But subsequent studies, specifically designed to evaluate palm oil, confirmed that palm oil's impact on serum lipid and lipoprotein profiles compares favorably to corn oil, lightly hydrogenated soybean oil, and olive oil. Thus, palm oil's impact on serum lipids is more like a monounsaturated than saturated oil. Palm oil contains a high percentage of monounsaturated (40\%). Palm oil's saturated fatty acids are palmitic (44\%) and stearic $(5 \%)$, which do not appear to evaluate blood cholesterol in people with cholesterol levels within normal ranges. Palm oil stimulates the synthesis of protective HDL cholesterol and removal of harmful Low Density lipoprotein (LDL) cholesterol. Palm oil is rich in vitamin $\mathrm{E}$, (particularly tocotrienols), which appear to reduce serum cholesterol concentrations (Ebong, et al. 1999). Hydrogenated vegetable fats are the problem; Animal fats are the problem.' Recently, an editorial by Harvard's Walter Willet, in the
American Journal of Public Health (1990) acknowledged that even though "' the focus of dietary recommendations is usually a reduction of saturated fat intake, no relation between saturated fat intake and risk of CHD was observed in the most informative prospective study to date." Another editorial, this time by Castelli, in the Archives of Internal Medicine (1992), declared for the record that "....in Mass, the more saturated fat one ate, the more cholesterol one ate, the more calories one ate, the lower the person's serum cholesterol. Would predict..." Castlli, (1992) further admitted that " for example, we found that the people who ate the most cholesterol, ate the most saturated fat, ate the most calories, weighed the least, and were the most physically active.',

Effects Of Palm Oil on Health: Beneficial substance can be detrimental in excessive quality. Malignant cells, on the other hand, are very sensitive to tocotrienols. In fact, the more cancerous the cell, the more susceptible it is to the destructive effects of tocotrienol, so very little is required to accomplish its favorable role of cancer cell annihilation (Ebong et al; 1999).

In developing countries, vegetable oils are replacing animal fats because of the cost and health concerns and Palm Oil has become one of the major edible oils in the world (Chandrasekharan, 1999). It is reassuring to know that the consumption of Palm Oil as a source of dietary fat does not pose any additional risks for coronary artery disease when consumed in realistic amounts as part of a healthy diet (Pedersen et al. 1999). Increasingly, over the past 40 years, the conception of diet has undergone major changes. Many of these changes involve changes of fats and oils. There has been an increasing supply of the partially hydrogenated trans- containing vegetable oils and a decreasing amount of the lauric acidcontaining oils (Enig, 1996). As a result, there has been an increased consumption of tran's fatty acids and linoleic acid and a decrease in the consumption of lauric acid. This type of change in diet has an effect on the fatty acids the body has available for metabolic activities. Although popular literature of epidemiological studies usually attribute an increased risk of coronary heart disease (CHD) to elevated levels of serum cholesterol, which in turn are thought to derive from a dietary intake of saturated fats and cholesterol (Mann, 1993).

Saturated fatty acids-; A considerable body of experimental has shown that blood cholesterol concentration can be modulated in individuals by alteration in the fatty acids content of the fat in their 
diet (Keys, 1965, Reiser, 1973). An increase in saturated fatty acid in the diet in the experiment generally leads to blood cholesterol content (LDL cholesterol). An average response of group of individuals is predictable to a reasonable approximation by the equations of Key's et al (Keys, 1965) or Hegsted et al (1965). Some saturated fatty acid are more effective than other (Bonanome, 1988), however, there is marked variation among individuals in the response observed (Ahrens, 1979). Because all naturally occurring fats and oil contains a range of fatty acids, experiment in this area can be interpreted. But it can be generally thought that the saturated fatty acid, stearic acid (18.0), when ingested as parts of a fat it does not tend to rise blood cholesterol concentration, whereas, palmitic acid (16.0) does. Stearic acid is a component of many fats, especially cocoa-butter, which is used in the chocolate production as palmitic acid is the most common fatty acid naturally found in fruits. Saturated fatty acids other then this two do not occur as commonly, it is thought that there is a generally effect of carbonchain length on the ability of a fatty acid to influence blood cholesterol concentration(Hegsted, 1965). Thus, the longer chain length fatty acid, with not less than or equal to 18 carbon atoms in the chain, seem to have little effect, whereas those medium length, with 10-16 carbon atoms, have a hypercholesterolemic effect. However, a recent careful study in monkeys suggested that palmitic acid have little effect when compared with the shorter chain length acid 12.0 (lauric acid), particularly, 14.0 (myrestic acid) (Hayes, et al; 1991).

Conclusion: Epidemiological studies usually attribute an increased risk of coronary heart disease (CHD) to elevated levels of serum cholesterol, which in turn are thought to derive from a dietary intake of saturated fats and cholesterol. Dietary fat is principally composed of triacylglycerol (TAG). Therefore, following digestion of a meal, there is a significant increase in the plasma TAG concentration. Prospective epidemiological studies show that plasma TAG, especially the no fasting level is an important factor in the pathogenesis of coronary heart disease. In the past, palm oil was attacked as "saturated" since it contains $44 \%$ palmitic acid and $5 \%$ stearic acid, and thereby allegedly raises blood cholesterol and increases the risk of cardiovascular disease. However, a sizeable and growing body of scientific evidence indicates that palm oil's effect on blood cholesterol is relatively neutral when compared to other fats and oils. Palm oil raises plasma cholesterol only when an excess of dietary cholesterol is presented in the diet. Palm oil stimulates the synthesis of protective HDL cholesterol and removal of harmful LDL cholesterol. Palm oil is rich in vitamin E, (particularly tocotrienols), which appear to reduce serum cholesterol concentrations and has potent anti-oxidant effects.

\section{REFERENCES}

Akintayo, E.T. and Bayer, E. (2002). Characterization and some possible uses of Plukenetia conophora and Adenopus breviflorus seeds and seed oils. Bioresource Technology 85: $95-97$.

Berger, K.G. (1992). Food uses of palm oil. Kuala Lumpur. Bulletin perkebunan, Vol.22:230-1

Castelli, W. P. (1992). Editorial: Concerning the possibility of a nut. Archives of Internal Medicine, 152: $1371-72$.

Chandrasekharan, N. (1999). Changing concepts in lipid nutrition in health and disease. Medical Journal of Malaysia, 54: 408 - 428.

Chandrasekharan, N.; Sundram, K.: Basiron, Y. (2000). Changing nutritional and health perspectives on palm oil. Brunei International Medical Journal, 2:417 - 427.

Chow, C.K. (1992). Fatty Acids in Foods and their Health Implications. New York: Marcel Dekker Inc., pp. $237-262$.

Clarke, R.; Frost, C.; Collins R.; Appleby, P.; Peto, R. (1997). Dietary lipids and blood cholesterol: quantitative meta-analysis of metabolic ward studies. British Medical Journal, 314: 112 - 117.

Cottrell, R.C. (1991). Introduction Nutritional aspects of palm oil. American journal of clinical nutrition 53: 989S - 1009S.

Ebong, P.E.; Owu, D.U.; Isong, E.U.; (1999). Influence of palm oil (Elaesis guineensis) on health. Plant Foods for Human Nutrition (Formerly Qualitas Plantarum), 53(3):209 - 222.

Enig, M.G.; (1996). Health and Nutritional Benefits from Coconut Oil: An Important Functional Food for the $21^{\text {st }}$ Century. Paper presented in the seminar AVOC Lauric Oils Symposium in Ho Chi Min City, Vietnam.

Enig, M.G.; (1993). Diet, serum cholesterol and coronary heart disease. In: GV Mann (Ed.): Coronary Heart Disease: The Dietary Sense and 
Nonsense. London: Janus Publishing, pp. 36 60.

Hornstra, (1990). Effects of dietary lipids on some aspects of the cardiovascular risk profile, In

Ziant, G. (Ed.): Lipids and Health: Proceedings of the $2^{\text {nd }}$ European Symposium on Lipids and Health: Lipids and Cancer, Lipids and Cardiovascular Diseases, Brussels, (International Congress Series). Amazon. Com. http://www.tocotrienol.org/en/index/news/58htm 1. http://www.bettykamen.com/newsletter /palmoil.htm.

http://www.tocotrienol.org/en/index/news/58html.

Institute of Medicine, National Academies (2002). Dietary Reference Intakes: Energy, Carbohydrate, Fiber, Fat, Fatty Acids, Cholesterol, protein, and Amino Acids (Macronutrients) pp. $11-17 . \quad$ National Academies Press, Washington DC.

Jensen, J.; Bysted, A.; Dawids, S.; Hermansen, K.; Holmer, G.; (1999). The effect of palm oil, lard, and puff-pastry margarine on postprandial lipid and hormone responses in normal weight and obese young women. British Journal of Nutrition, 82: 469 - 479.

Kheiri, S.A. (1987). End uses of palm oil: Human Food. In critical reports on applied chemistry, Vol. 15. Palm oil ed. F.D. Gunstone, $71-83$. London.

Koh, C.S. (2007). Comments on Draft Document: Diet, Nutrition, and the Prevention of Chronic Diseases.

Available in <http://www. who. int/dietphysicalactivity/media/en/gsfao_cmo_068

Mann, G.V. (1993). A short history of the diet heart hypothesis, In: GV Mann (Ed.): Coronary Heart Disease: The Dietary Sense and Nonsense. London: Janus Pulishing, pp. 1 - 17

Mensink, R.P.; Zock, P.L.; Kester, A.D.M.; Katan, M.B.; (2003). Effects of dietary fatty acids acids and carbohydrates on the ratio of serum total to HDL cholesterol and on serum lipids and apolipoproteins: a meta-analysis of 60 controlled studies. American Journal of Clinical Nutrition. 77: 1146-55.
Nagendran, U.R.; Unnithan, Y.M.; Choo, K.S. (2000). Food and Nutrition Bulletin, Vol. 21, (2) pg $77-82$. The United Nations University.

Pedersen, A.; Marckman, P.; Sandstrom, B. (1999). Post prandial lipoprotein, glucose and insulin response after two consecutive meals containing rapeseed oil, sunflower oil or palm oil with or without glucose at the first meal. British Journal of Nutrition, 82: 97 - 104.

Poku, K (2002). "origin of oil palm" small scale palm oil processing in Africa. FAO Agricultural Science Bulletin 148. Food and Agricultural Organisation.

Ravnskov, U. (1995). Quotation bias in reviews of the dietheart idea. Journal of Clinical Epidemiology, 48: 713:719.

Roche, H.M.; (2000) Low-fat diets, triglycerides and coronary heart disease risk. Nutrition Bulletin 25:49-53.

Rona, C.; Vailati, F.; Berardesca, E. (2004). 'Cosmetic treatment of wrinkles' Journal of cosmetic dermatology 3 (1): 26-34.

Smith, R.L. (1991). The Cholesterol Conspiracy St. Louis, Missouri: Warren H Green Inc.

WHO (2003). Diet Nutrition and the Prevention of Chronic Diseases. WHO Technical Report Series 916. Geneva: WHO, P, 82, 88 \& 88c.

Stuchlik, M.; Zak, S. (2002). 'Vegetable lipids as components of functional foods' Biomedical papers of the medical faculty of the university palacky, Olomouc, Czechoslovakia 146 (2) :3 10

Sutapa, M. and Analava, M. (2009). Health effects of Palm Oil. J Hum Ecol, 26(3): 197 - 203

Zhang, J,: Ping W.; Chunrong. W. Shou, X.C., Keyou, G (1997), Nonhypercholesterolemic Effects of a Plam Oil Diet in Chinese Adults. The Journal of Nutrition, 127: 509S-513S. 